\title{
Sclerosing angiomatoid nodular transformation of the spleen treated by laparoscopic partial splenectomy
}

\author{
Andrzej Budzyński', Sergiusz Demczuk ${ }^{2}$, Beata Kumiega ${ }^{3}$, Marcin Migaczewski ${ }^{1}$, Maciej Matłok ${ }^{1}$, Anna Zub-Pokrowiecka ${ }^{1}$ \\ ${ }^{1} 2^{\text {nd }}$ Department of General Surgery, Jagiellonian University Collegium Medicum, Cracow, Poland \\ 2Department of Pathology, Jagiellonian University Collegium Medicum, Cracow, Poland \\ 3Podkarpacki Centre of Oncology, Brzozów, Poland
}

Videosurgery and Other Miniinvasive Techniques 2011; 6 (4): 249-255

DOI: 10.5114/wiitm.2011.26261

\begin{abstract}
The authors describe a case of sclerosing angiomatoid nodular transformation (SANT) of the spleen treated at the $2^{\text {nd }}$ Department of Surgery, Jagiellonian University, Medical College. The patient was a 23-year-old woman. Clinically she presented with 2-year history of recurrent mild fever, diffuse joint pain, abdominal discomfort and iron deficiency anaemia of chronic disease. The laboratory tests revealed a non-characteristic chronic inflammatory response. A splenic solid lesion $9 \mathrm{~cm}$ in diameter was found on abdominal computed tomography. The patient underwent uneventful laparoscopic resection of the upper half of the splenic parenchyma. The resected tumour showed characteristic histological and immunophenotypical findings of SANT as previously described in the literature. In long-term follow-up, improvement of preoperative symptoms and abnormalities in the blood tests was documented.
\end{abstract}

Key words: sclerosing angiomatoid nodular transformation, sclerosing angiomatoid nodular transformation, spleen, laparoscopic partial splenectomy.

\section{Introduction}

Sclerosing angiomatoid nodular transformation (SANT) of the spleen is a rare benign vascular lesion, first described in the literature under this name in $2004[1,2]$. This rarely reported benign tumour is characterized by nodular vascular proliferation of the red pulp with prominent fibrosis [3]. Its aetiology is generally unknown. Most cases are either completely asymptomatic, an incidental finding or are associated with vague abdominal complaints. We report a case of SANT found during diagnostic workup in a patient with unexplained iron-deficiency anaemia and unspecific features of chronic inflammation.

\section{Case report}

The 23-year-old female patient was referred to the $2^{\text {nd }}$ Department of Surgery of Jagiellonian University, Medical College for treatment of tumour of the spleen. The patient presented with 2-year history of recurrent mild fever, and diffuse joint pain mostly of the small joints of the hand and spine. The patient also reported occasional vague abdominal discomfort, usually localized in the middle abdomen. The patient was lean (body mass index - BMI $17 \mathrm{~kg} / \mathrm{m}^{2}$ ) and the tip of the spleen was palpable during deep breathing. Apart from that her vital signs and physical examination were within normal limits. Her past medical and family history were also unremarkable. 


\section{Clinical features}

The laboratory tests revealed mild iron deficiency anaemia characteristic for chronic disease (RBC $4.22 \times 10^{12} / \mathrm{l}, \mathrm{HGB}-8.1 \mathrm{~g} / \mathrm{dl}, \mathrm{HCT}-27 \%, \mathrm{MCV}-64 \mathrm{fl}$, $\left.\mathrm{PLT}-458 \times 10^{9} / \mathrm{l}, \mathrm{WBC}-8.5 \times 10^{9} / \mathrm{l}\right)$. Apart from that, some features of a chronic inflammatory response were present, mainly represented by increased C-reactive protein (CRP) $(113.5 \mathrm{mg} / \mathrm{l})$ and fibrinogen $(534 \mathrm{mg} / \mathrm{dl})$ and a marked decrease of iron $(1.3 \mu \mathrm{mol} / \mathrm{l})$. The erythrocyte sedimentation rate (ESR) was increased (75/121). Other basic biochemical examinations were within normal limits. Bone marrow biopsy revealed mild stimulation of the granulopoietic cell lines with normoblastic erythropoiesis $(M: E=8: 1)$. Chronic blood loss through the gastrointestinal tract was ruled out by a negative test for occult blood in the stool, gastroscopy and colonoscopy. No pathology was found on gynaecological examination.

Tests for chronic infectious diseases (mononucleosis, CMV, hepatitis B, hepatitis C, borreliosis) were negative. Plasma antinuclear (ANA), antineutrophil cytoplasmic (ANCA) and endomysial antibodies in duodenal biopsy were not present.

Abdominal computed tomography (CT) and magnetic resonance imaging (MRI) revealed a well-circumscribed $8.5 \mathrm{~cm} \times 7.5 \mathrm{~cm} \times 9 \mathrm{~cm}$ tumour in the upper part of the spleen. The pattern of contrast enhancement suggested giant encapsulated cavernous haemangioma with pronounced fibrosis (Figure 1).

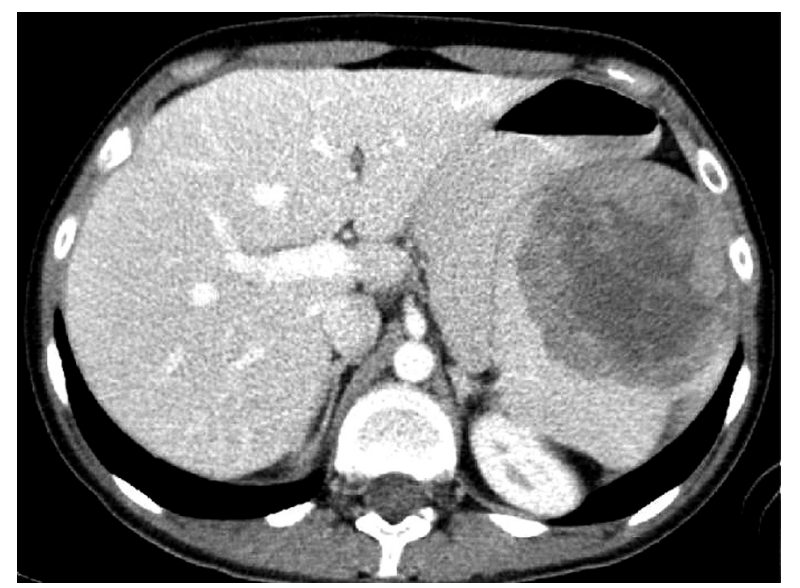

\section{Treatment}

The patient was submitted to laparoscopic partial splenectomy. The procedure was performed in the right lateral decubitus position. The pneumoperitoneum was established with the closed technique and 4 trocars along the left costal margin were inserted. The lesser sac was opened by transection of the gastrosplenic ligament using a harmonic scalpel and full mobilization of the spleen was achieved by dissection of its posterior attachments. The tumour was localized in the upper part of the spleen (Figure 2). The segmental branches of splenic vessels supplying the affected part of the organ were isolated and transected with Ligasure (Figure 3). Selective devascularization of the upper part of the spleen involving the macroscopically visible tumour was confirmed by the change in colour. The border of ischaemic tissue was marked by electrocautery. The remaining part of the vascular pedicle was temporarily closed with rubber band (Figure 4). In transient ischaemia splenic parenchyma was transected with Ligasure. The exposed splenic tissue at the resection line was coagulated with argon plasma coagulation (APC) (Figure 5). After removal of the rubber ligature the revascularization was confirmed by change in colour of the remaining part of the spleen and Doppler ultrasound was performed to document the restoration of normal blood flow (Figure 6).

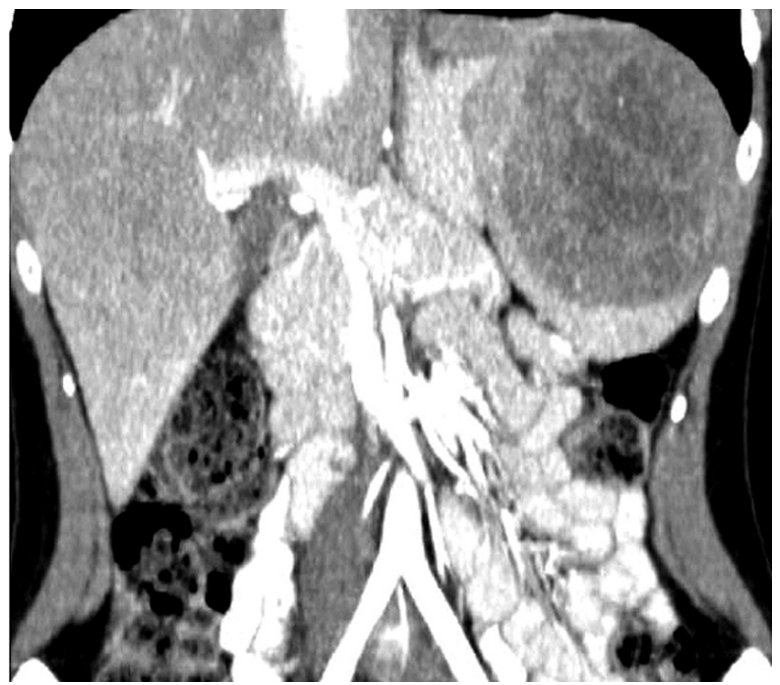

Figure 1. Well-circumscribed highly vascularised lesion with pronounced fibrosis in the upper part of the spleen found on abdominal CT 


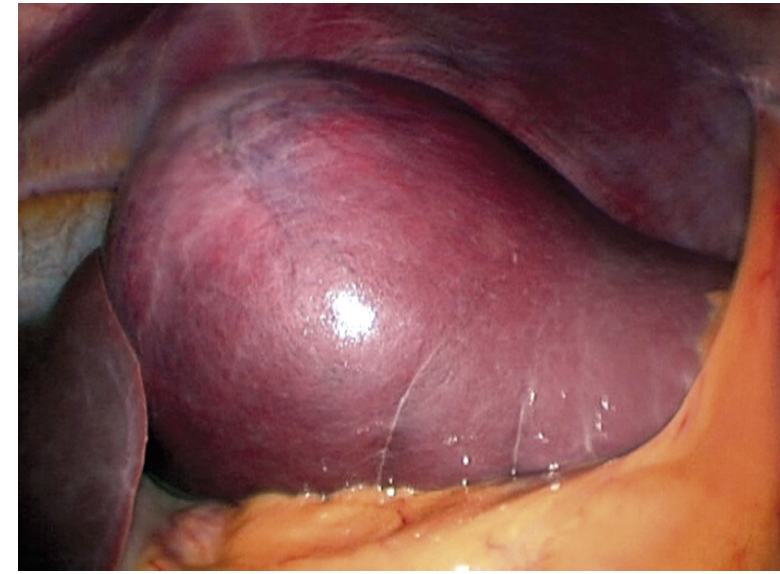

Figure 2. Solid tumour involving upper $2 / 3$ of the spleen viewed in laparoscopy
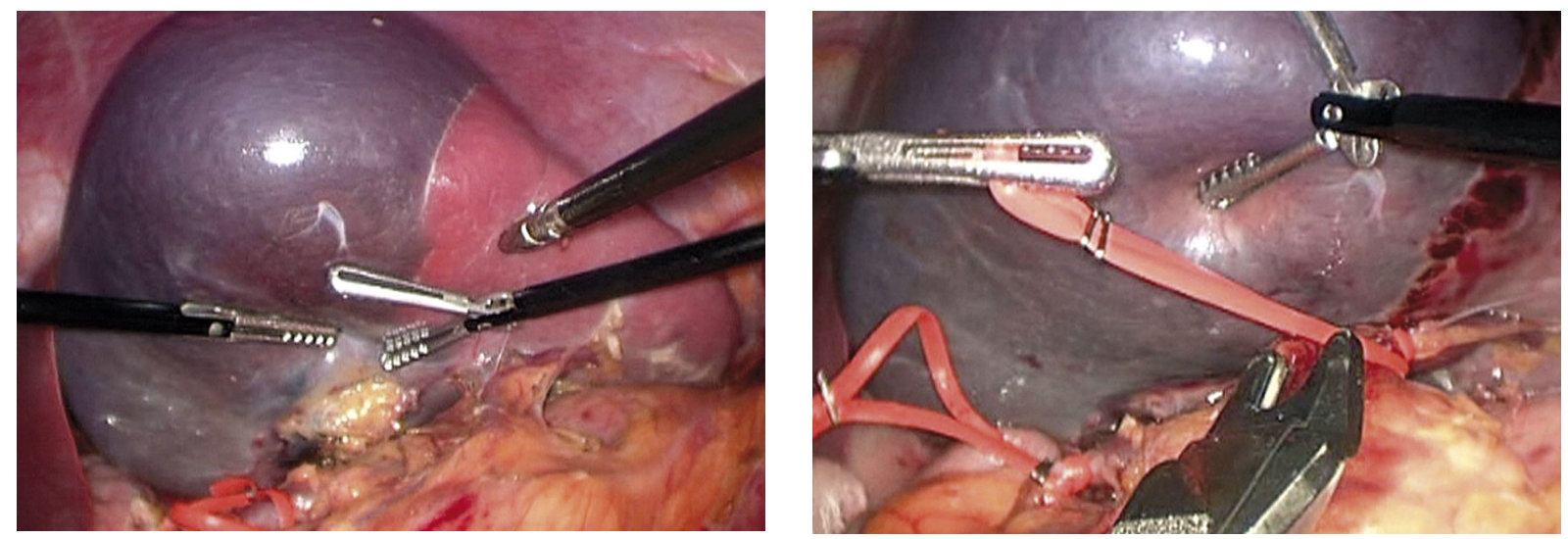

Figure 4. Partially devascularized upper $2 / 3$ of the spleen and temporal closure of the vascular pedicle supplying lower $1 / 3$ of the spleen with rubber band
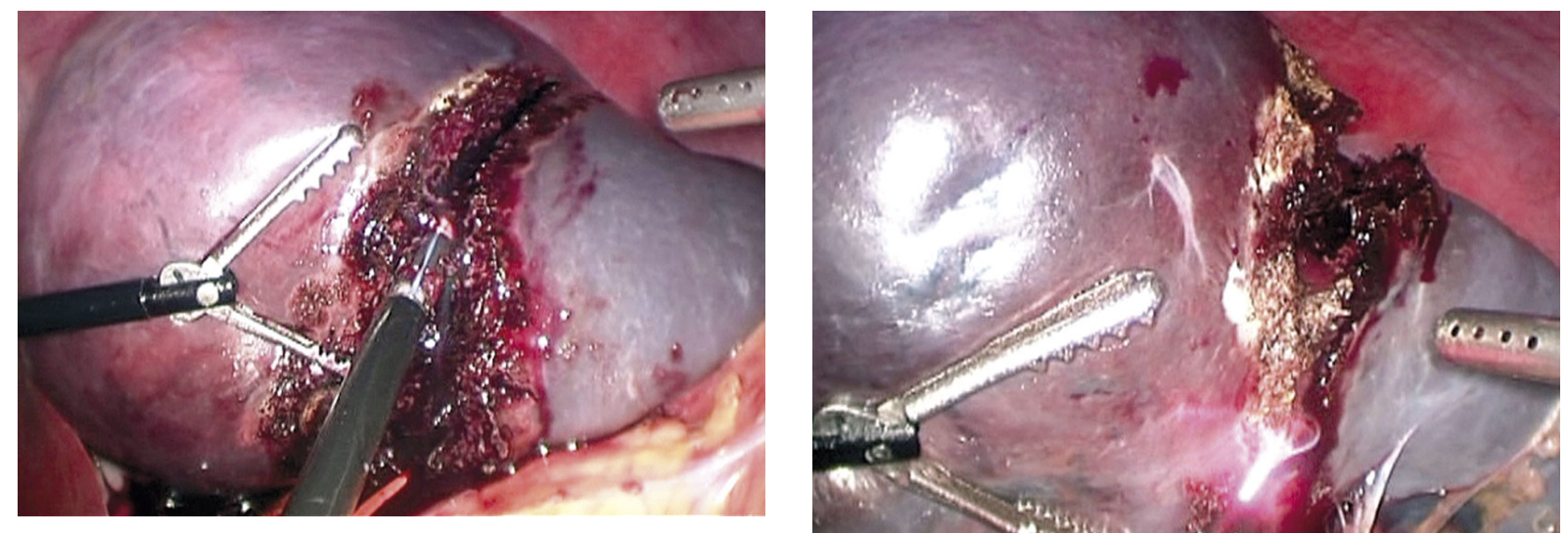

Figure 5. Resection of the splenic parenchyma with Ligasure and argon plasma coagulation 

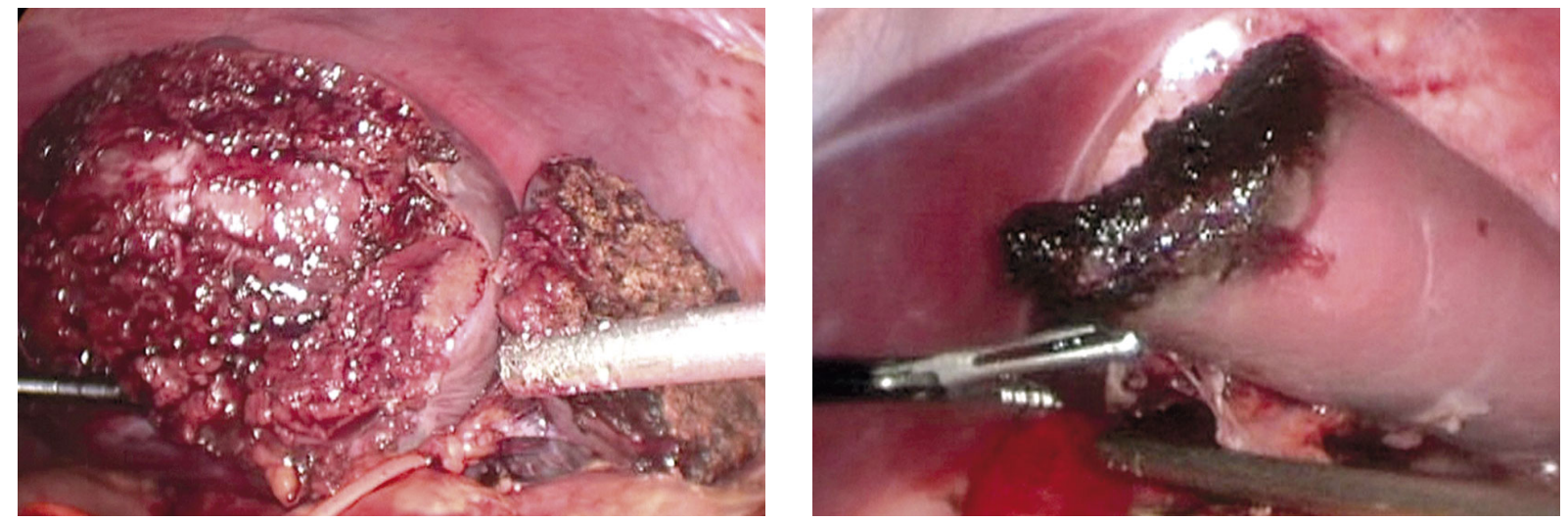

Figure 6. Completed resection of upper $2 / 3$ of the spleen and revascularization of the remaining part after removal of the rubber ligature

\section{Pathological findings}

The surgical specimen was evaluated in the Department of Jagiellonian University, Medical College. It consisted of spleen fragments weighing $266 \mathrm{~g}$ with a well-circumscribed unencapsulated mass. Microscopically the lesion comprised multiple variably sized angiomatoid nodules arranged in a lobular pattern (Figure 7). Each nodule was surrounded by thick fibrosclerotic stroma with a variable number of lymphocytes, plasma cells, macrophages and dispersed

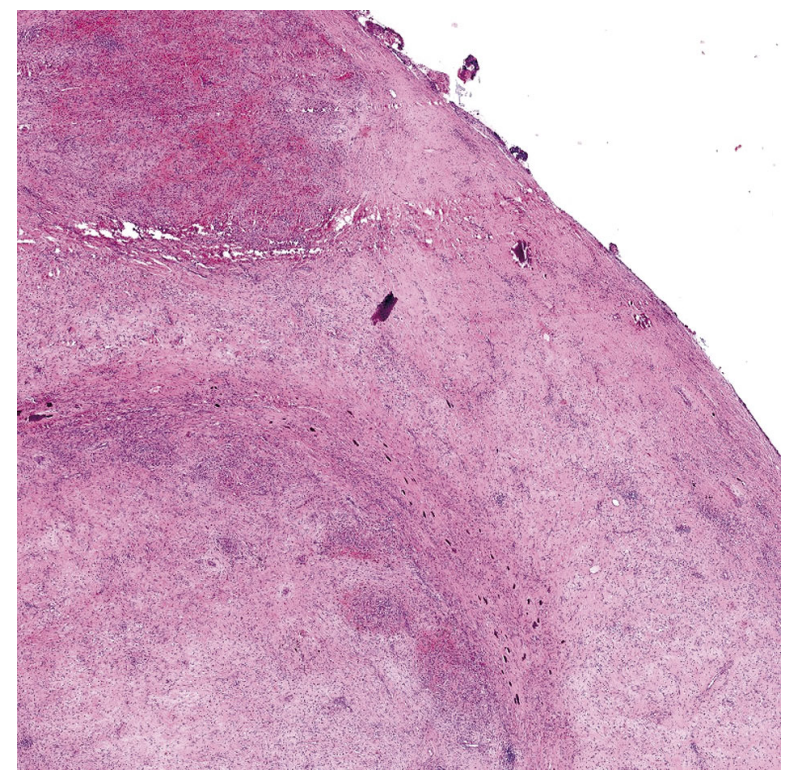

Figure 7. At low power magnification SANT is composed of multiple angiomatous nodules, some of them surrounded by hypocellular sclerotic stroma with focal calcifications (HE stain, Aperio ScanScope CS System) calcifications in some areas. Most of the nodules were composed of a haphazardly distributed sievelike complex of vascular spaces. Vascular spaces displayed capillaries, sinusoid-like spaces and small, venous-like channels that contained erythrocytes (Figure 8). Numerous cells in the angiomatoid nodules were CD31 positive (Figure 9) with only selective CD34 staining of the narrow capillaries (Figure 10).

\section{Follow-up}

One month after surgery the patient underwent complete diagnostic workup which revealed improvement in her anaemia (RBC - $4.94 \times 10^{12} /$, HGB -10.4 g/dl, HCT - 33\%, MCV - 66 fl, PLT $-345 \times 10^{9} /$, WBC $\left.-8.5 \times 10^{9} / \mu \mathrm{l}\right)$, and increase in iron level $(5.4 \mu \mathrm{mol} / \mathrm{l})$. Both CRP $(9.8 \mathrm{mg} / \mathrm{l})$ and fibrinogen $(384 \mathrm{mg} / \mathrm{dl})$ were

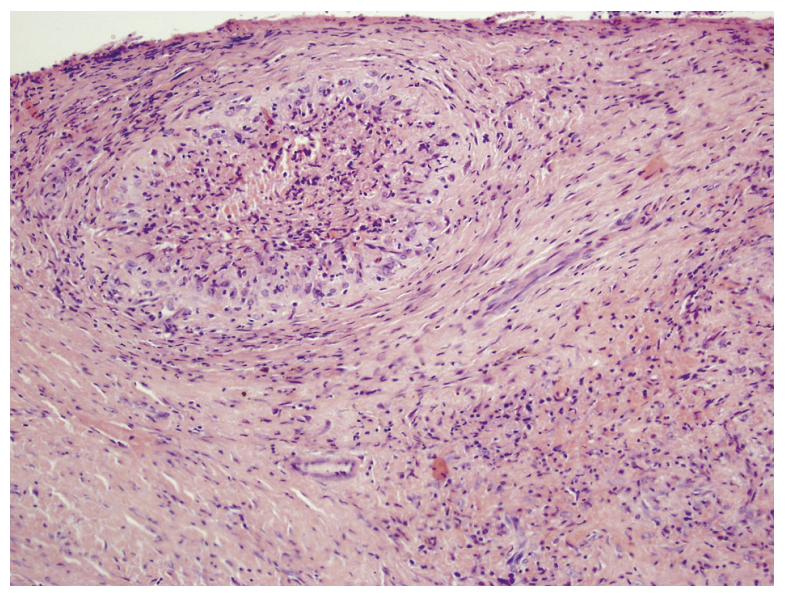

Figure 8. Angiomatoid nodules, some of them having a convoluted outline with surrounding concentric rings of collagen (HE stain) 


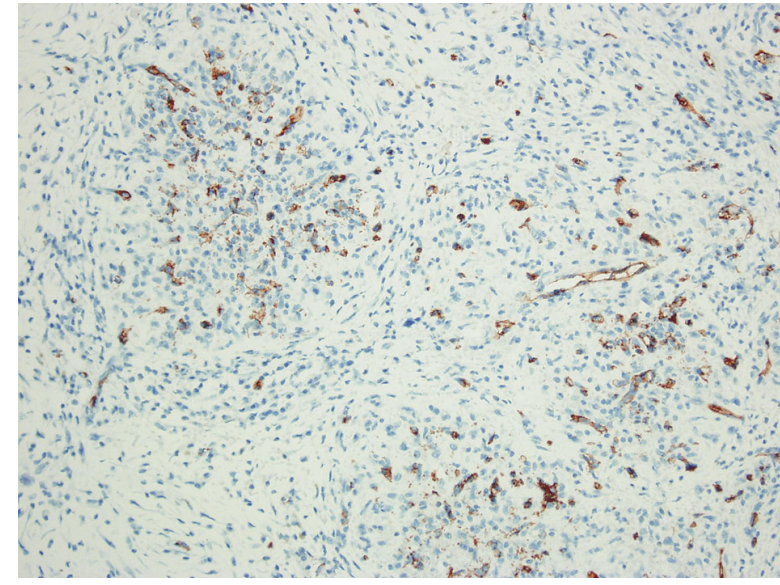

Figure 9. Positive staining for CD31 in the numerous cells of the angiomatoid nodules

within the normal range. The remaining part of the spleen showed some enlargement on abdominal ultrasound; its length was $6.5 \mathrm{~cm}$ and it has physiological blood flow documented by Doppler technique (Figure 11). Similar results were obtained at 1-year follow-up.

\section{Discussion}

Among isolated primary splenic disorders, approximately 50 cases of benign vascular lesion referred to as sclerosing angiomatoid nodular transformation (SANT) have been described. Formerly reported under different names as cord capillary haemangioma, splenic hamartoma, multinodular haemangioma or as a variant of splenic haemangioendothelioma, the lesion was finally designated in 2004 by Martel et al. [4, 5].

The most common presentations were incidental finding of an asymptomatic splenic mass, abdominal pain or discomfort, and splenomegaly. To the best of our knowledge, a clear association with other symptoms or complaints has not been described so far. In the original report of Martel describing the largest series so far, one out of 25 patients presented with leukocytosis, polyclonal gammopathy, and raised erythrocyte sedimentation rate (ESR), one with fever, one with mild anaemia, and another one with pancytopenia with elevated ESR. More clinical data are given in the Diebold study [6]. Three out of 16 patients in his study had some inflammatory condition and other 3 had anaemia.

The unique feature of our case was a generalized inflammatory reaction manifested by fever, muscular

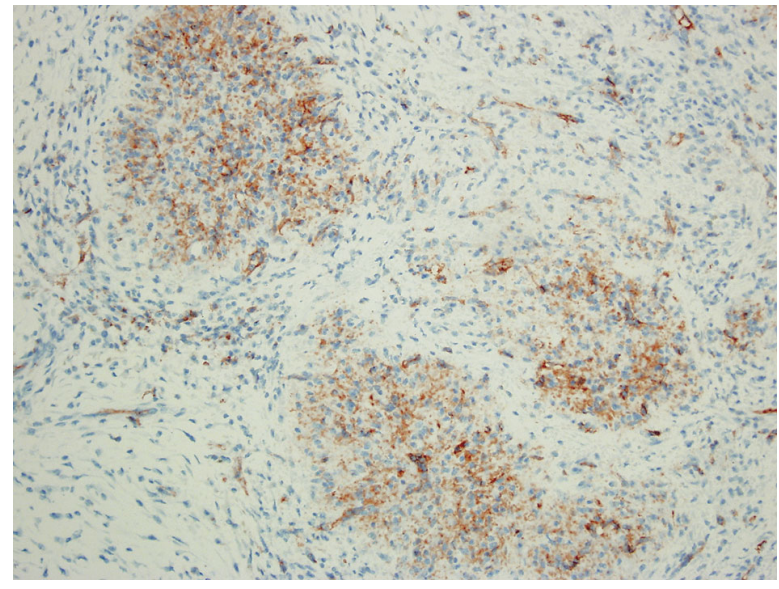

Figure 10. Positive CD34 staining only selective of the narrow capillaries

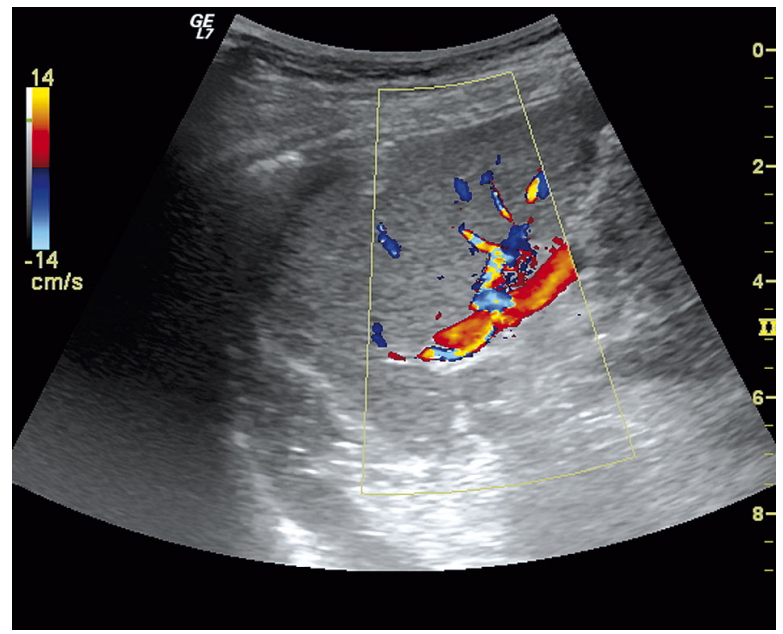

Figure 11. Follow-up ultrasound showing normal flow pattern through the fragment of the spleen left after partial splenectomy

and joint pain, elevation of ESR, CRP and fibrinogen, and iron-deficiency anaemia. This clinical picture necessitated a diagnostic workup in the search for a possible explanation, and as a final result the tumour of the spleen was found. This history is somewhat distinct from previous reports. The radiographic, gross, histological, and immunophenotypical features were similar to the cases previously described in the literature.

The main differential diagnoses of SANT include littoral cell angioma, splenic haemangioendothelioma, inflammatory myofibroblastic tumour, splenic hamartomas and nodular transformation of splenic red pulp in response to metastatic carcinoma [7-13]. 
Others are congestive splenomegaly, infarction and Kaposi's sarcoma [14].

The clinical features present in our case support the assumption that there might be some inflammatory component in the aetiology of this disease. According to different authors, some lesions may represent some form of inflammatory pseudotumours, hamartomatous lesions, red pulp transformation in response to exaggerated stromal proliferation or organized haematomas [15-17]. The theory about inflammatory components in the aetiology could be supported by complete disappearance of the biochemical indicators of inflammation after surgical removal of the tumour.

In practice, highly vascularised lesions in the splenic parenchyma are very difficult to differentiate preoperatively [18]. The most common pathological finding is haemangioma, but other tumours such as splenic hamartoma, haemangioendothelioma, littoral cell angioma, benign vascular tumours with myoid and angioendotheliomatous features, inflammatory myofibroblastic tumour (inflammatory pseudotumour) or hamartomas are occasionally found $[6,19-21]$. Usually the only method to establish an unequivocal diagnosis is pathological examination of the spleen, and splenectomy is considered both diagnostic and curative. The main reason for surgical management is uncertainty about the nature of the tumour of the spleen. This approach is particularly supported by the fact that many patients with SANT have a history of previous malignancy, and splenic tumour is frequently found during routine follow-up in the search for metastases. Moreover, clinical and radiological features of the SANT may mimic nodular transformation of the splenic red pulp in response to metastatic carcino$\mathrm{ma}$, and important risk of this pathology is the strongest indication for splenectomy.

In all reports, splenectomy is considered curative with regard to the risk of recurrence. However, this treatment is associated with all the long-term risks related to asplenia. Most important seems the overwhelming post-splenectomy infection (OPSI) with the high mortality rate related to this disease entity. Benign nature of the tumour in various imaging techniques including $\mathrm{CT}, \mathrm{MRI}$ or positron emission tomography (PET) scans allows for a different surgical approach. Whenever technically possible, partial splenectomy with adequate margins of uninvolved tissue seems appropriate. With high experience in advanced minimally invasive procedures the laparoscopic approach is considered beneficial [22, 23].

Owing to the extensive experience in laparoscopic operation of the spleen, we may conclude that even though indications for spleen preserving procedures are scarce, they are highly beneficial for the patient. If the vascular anatomy and localization of the lesion allow for the selective devascularization of the affected part of the spleen, the SANT tumour could be considered for laparoscopic hemisplenectomy. Generally, the best indications for this advanced procedure are splenic cysts and tumours with benign radiological phenotype localized in the upper or lower part of the spleen. Central localization of the lesion usually does not allow for partial splenectomy.

Relatively high prevalence of malignancy among patients with SANT was described. However, further studies are needed to conclude whether it could be classified as paraneoplastic syndrome or it is just the result of frequent use of the variety of imaging studies in oncological patients that lead to the detection of various asymptomatic lesions. So far - as long as the data about the relationship between SANT and malignancy is not clear - regular follow-up should be recommended in all patients with confirmed SANT.

\section{References}

1. El Demellawy D, Nasr A, Alowami S. Sclerosing angiomatoid nodular transformation of the spleen: case report. Pathol Res Pract 2009; 205: 289-93.

2. Koreishi AF, Saenz AJ, Fleming SE, Teruya-Feldstein J. Sclerosing Angiomatoid Nodular Transformation (SANT) of the spleen: a report of 3 cases. Int J Surg Pathol 2009; 17: 384-9.

3. Chouchane Mlik O, Lardenois S, Salmon R, et al. Sclerosing angiomatoid nodular transformation of the spleen. Ann Pathol 2008; 28: 317-20.

4. Lee JC, Lien HC, Hsiao CH. Coexisting sclerosing angiomatoid nodular transformation of the spleen with multiple calcifying fibrous pseudotumors in a patient. J Formos Med Assoc 2007; 3: 234-9.

5. Martel M, Cheuk W, Lombardi L, et al. Sclerosing angiomatoid nodular transformation (SANT): report of 25 cases of a distinctive benign splenic lesion. Am J Surg Pathol 2004; 28: 1268-79.

6. Diebold J, Le Tourneau A, Marmey B, et al. Is sclerosing angiomatoid nodular transformation (SANT) of the splenic red pulp identical to inflammatory pseudotumour? Report of 16 cases. Histopathology 2008; 53: 299-310.

7. Ali TZ, Beyer G, Taylor M, et al. Splenic hamartoma: immunohistochemical and ultrastructural profile of two cases. Int J Surg Pathol 2005; 13: 103-11.

8. Arber DA, Strickler JG, Chen Y, et al. Splenic vascular tumors: a histologic, immunophenotypic, and virologic study. Am J Surg Pathol 1997; 21: 827-35. 
9. Daze Y, Gosselin J, Bernier V. Sclerosing angiomatoid nodular transformation of the spleen (SANT): a case report. Ann Pathol 2008; 28: 321-3.

10. Fakan F, Michal M. Nodular transformation of splenic red pulp due to carcinomatous infiltration. A diagnostic pitfall. Histopathology 1994; 25: 175-8.

11. Falk S, Stutte HJ, Frizzera G. Littoral cell angioma. A novel splenic vascular lesion demonstrating histiocytic differentiation. Am J Surg Pathol 1991; 15: 1023-33.

12. Kaw YT, Duwaji MS, Knisley RE, et al. Hemangioendothelioma of the spleen. Arch Pathol Lab Med 1992; 116: 1079-82.

13. Li L, Fisher DA, Stanek AE. Sclerosing angiomatoid nodular transformation (SANT) of the spleen: addition of a case with focal CD68 staining and distinctive CT features. Am J Surg Pathol 2005; 29: 839-41.

14. Cook PD, Czerniak B, Chan JK, et al. Nodular spindle-cell vascular transformation of lymph nodes. A benign process occurring predominantly in retroperitoneal lymph nodes draining carcinomas that can simulate Kaposi's sarcoma or metastatic tumor. Am J Surg Pathol 1995; 19: 1010-20.

15. Falk S, Stutte HJ. Hamartomas of the spleen: a study of 20 biopsy cases. Histopathology 1989; 14: 603-12.

16. Neuhauser TS, Derringer GA, Thompson LD, et al. Splenic inflammatory myofibroblastic tumor (inflammatory pseudotumor): a clinicopathologic and immunophenotypic study of 12 cases. Arch Pathol Lab Med 2001; 125: 379-85.

17. Seijo L, Unger P, Strauchen J. Inflammatory pseudotumor of the spleen: an old concept with many questions. Int I Surg Patho 1996; 43: 289-98.

18. Kutok JL, Fletcher CD. Splenic vascular tumors. Semin Diagn Pathol 2003; 20: 128-39.

19. Karim RZ, Ma-Wyatt J, Cox M, Scolyer RA. Myoid angioendothelioma of the spleen. Int J Surg Pathol 2004; 12: 51-6.

20. Kraus MD, Dehner LP. Benign vascular neoplasms of the spleen with myoid and angioendotheliomatous features. Histopathology 1999; 35: 328-36.

21. Szpor J, Dyduch G. From hamartoma to splenic hemangioma. Pol J Pathol 2008; 59: 33-41

22. Bednarek M, Budzyński P, Budzyński A, Demczuk S. Sarcoidosis of the spleen - rare indications for splenectomy. Own experience. Videosurgery and Other Miniinvasive Techniques 2010; 5 : 115-9.

23. Dzielicki J, Grabowski A, Korlacki W. Optimizing the technique of laparoscopic splenectomy in children. Videosurgery and Other Miniinvasive Techniques 2010; 5: 19-26. 